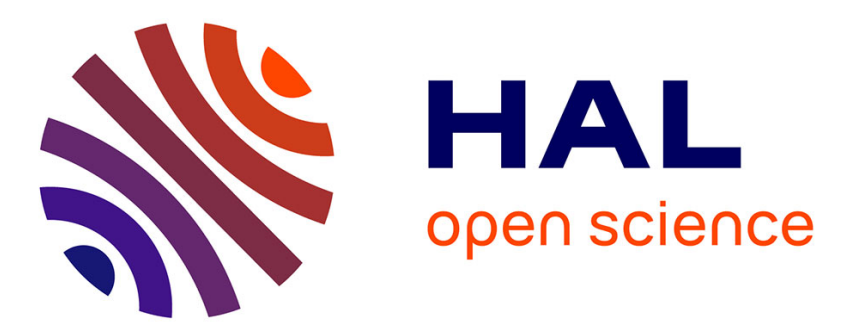

\title{
A Review of Haptic Feedback Teleoperation Systems for Micromanipulation and Microassembly
}

\author{
Aude Bolopion, Stéphane Régnier
}

\section{To cite this version:}

Aude Bolopion, Stéphane Régnier. A Review of Haptic Feedback Teleoperation Systems for Micromanipulation and Microassembly. IEEE Transactions on Automation Science and Engineering, 2013, 3, pp.496-502. hal-00841740

\author{
HAL Id: hal-00841740 \\ https://hal.science/hal-00841740
}

Submitted on 5 Jul 2013

HAL is a multi-disciplinary open access archive for the deposit and dissemination of scientific research documents, whether they are published or not. The documents may come from teaching and research institutions in France or abroad, or from public or private research centers.
L'archive ouverte pluridisciplinaire HAL, est destinée au dépôt et à la diffusion de documents scientifiques de niveau recherche, publiés ou non, émanant des établissements d'enseignement et de recherche français ou étrangers, des laboratoires publics ou privés. 


\title{
A Review of Haptic Feedback Teleoperation Systems for Micromanipulation and Microassembly
}

\author{
Aude Bolopion ${ }^{1}$ and Stéphane Régnier ${ }^{2}$
}

\begin{abstract}
This paper presents a review of the major haptic feedback teleoperation systems for micromanipulation. During the last decade, the handling of micrometer-sized objects has become a critical issue. Fields of application from material science to electronics demonstrate an urgent need for intuitive and flexible manipulation systems able to deal with small-scale industrial projects and assembly tasks. Two main approaches have been considered: fully automated tasks and manual operation. The first one require fully pre determined tasks, while the later necessitates highly trained operators. To overcome these issues the use of haptic feedback teleoperation where the user manipulates the tool through a joystick whilst feeling a force feedback, appears to be a promising solution as it allows high intuitiveness and flexibility. Major advances have been achieved during this last decade, starting with systems that enable the operator to feel the substrate topology, to the current state-of-the-art where 3D haptic feedback is provided to aid manipulation tasks. This paper details the major achievements and the solutions that have been developed to propose 3D haptic feedback for tools that often lack 3D force measurements. The use of virtual reality to enhance the immersion is also addressed. The strategies developed provide haptic feedback teleoperation systems with a high degree of assistance and for a wide range of micromanipulation tools. Based on this expertise on haptic for micromanipulation and virtual reality assistance it is now possible to propose microassembly systems for objects as small as 1 to 10 micrometers. This is a mature field and will benefit small-scale industrial projects where precision and flexibility in microassembly are required.
\end{abstract}

\section{Note to Practitioners}

This paper is motivated by the urgent need of intuitive and flexible manipulation systems able to deal with assembly tasks on the microscale. A new and promising solution is presented here; teleoperation with force feedback, where an operator uses a joystick to control the tool at the microscale, whilst experiencing interaction forces between the tool and the environment. Feedback assistance to the user using attractive and repulsive force fields is also be proposed to help achieve the assembly task. Examples are given in the paper to illustrate this approach. The presented techniques can be readily applied to most micromanipulation systems to perform advanced microassembly tasks.

Index Terms-Haptic feedback, Teleoperation, Micromanipulation, Microassembly

\section{INTRODUCTION}

During the last decade, interest in microassembly has increased dramatically across a wide range of application fields

\footnotetext{
1 Institut FEMTO-ST, UFC, ENSMM, UTBM, CNRS UMR 6174, 24 rue Alain Savary, 25000 Besançon, France. aude.bolopionefemto-st. fr

2 Institut des Systèmes Intelligents et de Robotique, Université Pierre et Marie Curie, CNRS UMR 7222, 4 Place Jussieu, 75005 Paris, France. regnierdisir.upmc.fr

Manuscript received; revised.
}

from material science to electronics [1]. Robotic devices based on: thermal actuation, shape-memory alloys, and piezoelectric or electrostatic principles have been developed to enable precise movements [2]. Several tools have been proposed for performing 3D operations, such as cantilevers and grippers [3], [4]. Automated tasks have been implemented for the assembly of objects the size of hundreds of micrometers [5]. However, this solution is valid only in a highly controlled environment [6], which limits the industrial applications because it makes the operation time-consuming and often expensive. In addition, it must be applied to large-scale projects where a given predefined task must be performed which is often not feasible when dealing with the assembly of novel products, such as innovative MOEMS (Micro Opto Electro Mechanical Systems) or MEMS (Micro Electro Mechanical Systems). On the microscale, most of the projects are smallscale industrial development or novel protocols. Proposing a fully automated setup for each assembly required can be time-consuming or even inefficient, as most of the protocols are not completely defined before the operation. The user's expertise and capacity to adapt the manipulation protocol to environmental disturbances and the peculiarities of the task is of the utmost importance to ensure the success of the assembly. Teleoperated tasks, where an operator manually performs the assembly by controlling the robotic system through a joystick, is thus a widely used solution [7]. However, only highly skilled operators can perform complex assemblies because the objects and tools are fragile, the systems are highly sensitive to environmental conditions, and the visual feedback is limited. Therefore, assistance must be provided in order to enable a higher number of users to perform the operations. Haptic feedback entails providing operators with a force feedback through the control joystick used for the manipulation (Fig. 1) and is a promising solution [8], [3]. A the microscale both theoretical works [9], [10], [11] and studies on systems dedicated to micromanipulation [12], [13], [14] have been conducted.

This article presents a review of the main haptic feedback teleoperation systems for micromanipulation. The major issues that must be addressed in order to obtain a usable system for small scale industrial projects are highlighted, and the solutions proposed in the literature are presented. In particular, the problems induced by high scaling factors and time delays on the performance of haptic coupling schemes are addressed. Solutions are proposed for providing haptic feedback on sensor deprived systems or systems where only part of the information is available. Examples of haptic feedback rendering are provided for classical micromanipulation tasks, such as 
pushing or pick-and-place. Additional methods of assistance using virtual reality are presented.

This paper is organized as follows. In Sec. II the major historical teleoperation systems are reviewed. The issues that must be faced in order to improve the performance of these early systems are presented in Sec. III, and solutions developed in the literature are reviewed. Haptic feedback teleoperation systems for micromanipulation tasks are given in Sec. IV, and Sec. V presents solutions incorporating additional assistance. Sec. VI concludes the paper.

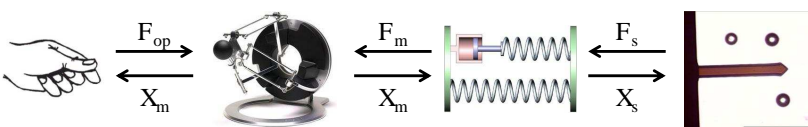

Fig. 1. Haptic feedback teleoperation system. The operator control the position of the tool $X_{s}$ by controlling the position of the haptic device handle $X_{m}$. Interaction forces applied on the tool are transmitted to the user as a haptic force $F_{m}$.

\section{Historical Teleoperation Systems}

The first use of teleoperation systems for micro and nano scales applications was published in [15] in 1990. The goal was to make a system able to reproduce the movements of the operator and scale them down to control a manipulator. It should also be able to reproduce the phenomena occurring at the microscale through visual and haptic feedback. However, this first implementation only gave access to a visual feedback, and information about forces was given by visual indications, not haptically. The first teleoperation system with haptic feedback appears in [16]. The haptic device is linked to a scanning tunneling microscope. Users control the in-plane displacement of the tip of the microscope. The vertical movement of the handle of the haptic device follows the vertical movements of the tip so that users can "feel" the topology of the substrate. However, a lot of noise and hysteresis limits the possible applications.

Since these two first works, teleoperation systems with haptic feedback have been mainly developed for AFM-based manipulation (Fig. 2). Two main reasons justify this choice: the Atomic Force Microscope is one of the most commonly used tools for manipulation of micron-sized objects and, more importantly, it is one of the few that enable force measurement, which is a critical issue to provide haptic feedback. The first system is presented in [17]. Only one degree of freedom is available, and users control the in-plane position of the tip using a mouse. They can feel the repulsive forces when a force is applied on the substrate by the tip, as well as the attractive forces when the tip is lifted away from the substrate [18]. Simple experiments have also been performed in [19]. In particular, tasks of picking up spheres by adhesion and releasing them by rolling have been realized. Only vertical forces are transmitted, and the use of a piezoresistive sensor limits the resolution of the measured force.

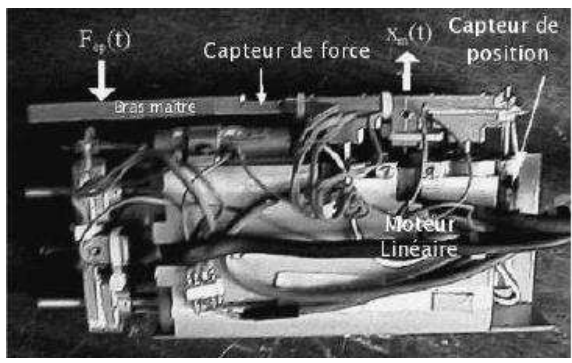

Fig. 2. Haptic feedback teleoperation systems, Institute of Industrial Sciences, Tokyo [20].

These first systems makes it possible to "touch the microworld", but not to feel interaction forces between the tool and an object, such as the grasping force applied by a gripper. They are thus not effective at performing manipulation or assembly tasks with controlled interaction force. The reasons for these limitations are detailed in the next section.

\section{MAJOR IsSUES}

The limited application of these systems is due to two main reasons. The first one is the stability issue. The scaling factors introduced to match variables in the macro and the micro worlds introduce instabilities. In addition, time delays that occur while dealing with simulated environments or when using vision sensors to compute the haptic feedback induce instabilities. The second reason is the incomplete force measurement. The force applied on the tool is most of the time deduced from the measurement of the tool's deformations. However, it is usually limited to 1D or 2D force sensing, depending on the geometry of the tool, and it provides only limited information on the interaction force between the tool and the object.

\section{A. Stability for High Scaling Gains Coupling Schemes and Time Delayed Systems}

A detailed analysis of the control schemes is of the utmost importance to provide a haptic feedback of good quality. This feedback should be stable, or else the user will need to compensate for large oscillations of the joystick that are disturbing, and might cause damage to either the haptic device or the tool. It should also be transparent, which means that the user can feel with a high degree of fidelity the interaction forces applied on the tool.

The control scheme depicted in Figure 3 is the most intuitive formulation to provide amplified forces to the operator [21]. Basically, the user operates a haptic device in the macro-world by applying a force $F_{o p}$ to impose the displacements of the slave device in the micro-world (velocity $V_{n}$ ). The velocity of the haptic device is scaled down by $\alpha_{p}$ to be used as the input of the nanotranslator. The force applied on the tool $F_{s}$ is amplified by $\alpha_{F}$ so that $F_{m}$ is sent to the user as the haptic feedback.

Solutions proposed for macro-sized systems are largely used to ensure stability, such as in [19] where the Llewelyn criterion [22] is applied. However, the solutions proposed for 


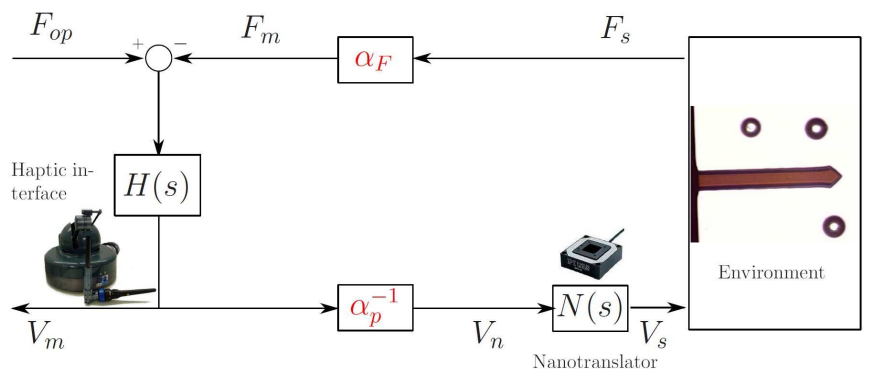

Fig. 3. Direct Force Feedback coupling scheme. Adapted from [21].

macro-sized systems may not be adapted to the specificities of the microworld. [23] presents an adapted passivity controller that enables users to feel attractive forces. It is first tested through simulations, before [24] demonstrates its application on a real system. The homothetic factors play a major role in the stability of the coupling schemes [17], [18]. A detailed analysis is provided in [21], which states rules to tune the coupling parameters to ensure stability and transparency. In particular, it highlights that a large amplification of the forces or large displacements (i.e., a large force scaling factor $\alpha_{F}$ or small displacement scaling factor $\alpha_{p}$ ) might induce instabilities. The ratio of the scaling factors must be less than a factor that depends on the haptic interface and the nanotranslator characteristics.

In addition to high scaling factors, a major source of instability is time delay. This issue becomes critical for applications where long-distance teleoperation is involved, or for interaction with complex virtual scenes or when visionbased sensors with slow acquisition time are used to compute haptic feedback. This issue is highly critical since at this scale objects experience high accelerations due to their small inertia. In addition the high sensibility of these systems to environmental conditions makes the prediction of the position of the manipulated object difficult. The stability of the system despite delays and modeling uncertainties is studied in [25] and [26], which respectively propose a wave variable and a $H_{\infty}$ controller. However the degradation of the transparency while implementing these control laws is a critical issue. Strategies to limit the time delays and acquisition time of the sensors are necessary. In particular high speed vision sensing is presented in the next sections of this paper.

\section{B. Limited Position and Force Measurement}

The second major issue is the limited position and force measurement. The development of such systems faces a major obstacle: the lack of position and force feedback [2]. Sensors have been developed [27], [28], but their integration into dedicated tools increases significantly the complexity and the cost of the tool fabrication.

AFM have been the first tools used for haptic feedback on the microscale since they enable force measurement. However, since forces are computed from the measurement of the cantilever's deformations, only two measures are available: the vertical bending and the torsion [29]. To improve the haptic feedback, [30] analyzes the relation between the threedimensional force applied on the cantilever and the measure of the deformations by taking into account the direction of the cantilever movement. However, this technique is highly sensitive to the noise measurement and numerical errors during the computation of the force.

Another approach is proposed in [31], which uses a model of friction between the tip and the substrate. The topology of the substrate is assumed to be known, for example from previous AFM scans. This solution is promising to provide users with information about the substrate, but it cannot be used for manipulations, as the interaction force between the object and the tool cannot be determined.

In addition to AFM, more complex tools have been developed to perform advanced assembly tasks. In particular, microgrippers are commonly used [1]. Even if some of them offer sensing capabilities (at the expense of a complex design) [32], [33], most still lack force measurement capabilities [34], [35]. In particular, only a few grippers enable the manipulation of objects of less than $10 \mu \mathrm{m}$ with force feedback, and most of them are only prototypes.

To overcome the lack of force-sensing, vision is a promising solution [36], [37]. It is used to measure the deformations of the tool [38]. Force is then estimated based on the mechanical properties of the tool [39], [40]. This solution avoids the complexity of force sensor integration, while providing feedback to compute the haptic force. Strategies based on virtual guides, using simulators or not, have also been developed to compensate for the limited position and force measurement. They can be used, for example, for micromanipulation under SEM (Scanning Electron Microscopes), where closed loop positioning tools are either expensive or have a limited bandwidth. In this case, the vision-based haptic feedback enables users to close the loop, and compensate for the lack of closed loop positioning units. This technique is used in some of the applications detailed in the next sections.

\section{Haptic FeEdback fOR Micromanipulation TASKs}

\section{A. Haptic Feedback of Nanonewton Interaction Forces}

Using AFM, the laser reflected on the cantilever makes it possible to measure the forces applied on the tool and to get a nanonewton force resolution measurement [41]. With an appropriate haptic coupling scheme, it is thus possible to render to operators very weak interaction forces, such as attractive forces between the substrate and a cantilever [21]. In particular, the snap-in phenomenon occurring while approaching the tip from the substrate, which is in the order of the $\mathrm{nN}$, is transmitted to users (Fig. 4). These systems enable a better comprehension of interactions between the objects, and in particular the influence of the nature of the substrate on the attractive force [24].

\section{B. Virtual Guides}

Instead of transmitting haptic forces that perfectly match measured forces, it can be interesting to define haptic forces 


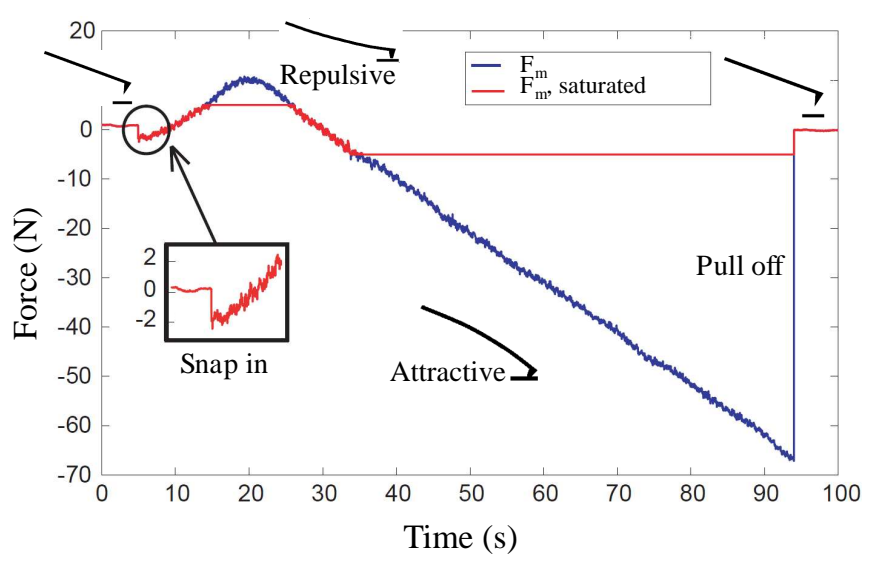

Fig. 4. Haptic feedback of an approach-retract experiment. The cantilever is approached from the substrate (time $t<20 \mathrm{~s}$ ). Users can feel interaction forces, and in particular the snap-in phenomenon (in the order of the nanonewton). When contact is reached, a force is applied on the substrate by the cantilever and users feel a repulsive force. The cantilever is then moved away from the substrate. Users feel attractive forces until the cantilever is detached from the substrate - adapted from [21]

that will help users to perform a given task. These virtual guides are used either to "pull" users into what is assumed to be the correct position, or to "push" them away from areas where the tool should not go, for example, to avoid collisions [42]. The user is thus guided, but he/she can decide to override this indication by applying a force greater than the haptic force on the joystick handle. The definition of these virtual guides is highly related to the task that must be done, and to the available position or force measurement.

Virtual guides dedicated to rolling tasks are demonstrated in [43], where 2D haptic feedback is provided. They assist users to keep the sphere under the middle line of the cantilever and at its extremity by providing them with information about the position of the object under the cantilever (Fig. 5). In [44], the two possibilities (faithful rendering of interaction forces or virtual guides) are proposed to pick up and place a microsphere. Virtual guides assist users to pull the sphere until the desired altitude and a repulsive force avoids any involuntary collision with the substrate.

However, it is not straightforward to determine which haptic feedback (faithful rendering of interaction forces or virtual guides) is the best adapted. This depends on the goal of the teleoperation. If a better comprehension of haptic phenomena is wanted, a faithful rendering is helpful, whereas to perform a given manipulation task virtual guides prove to be effective.

\section{Haptic Feedback for Systems with Limited Position and Force Sensing Capabilities}

Some works have been proposed to use two AFM cantilevers with protrudent tips to make a gripper [45]. To detect the position of the object, the cantilevers are used in dynamic mode to ensure accurate measurements [46]. The cantilevers are excited at their resonant frequency, and the amplitudes of the oscillations are measured. Adapted haptic feedback based

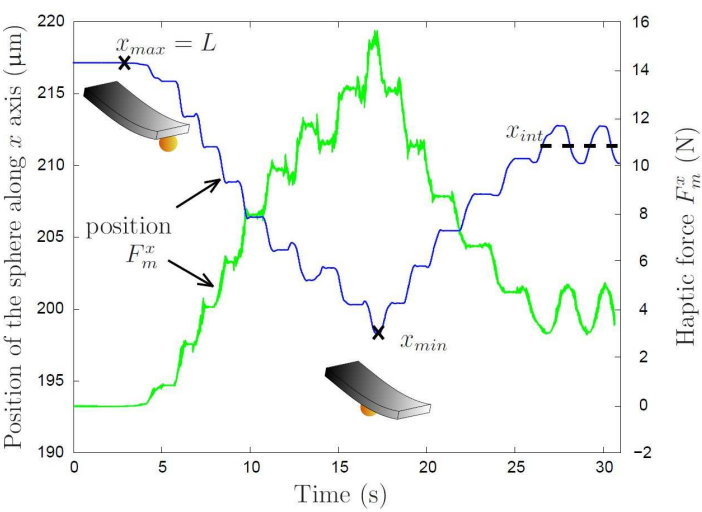

Fig. 5. Virtual guide that assists the user while keeping the sphere at the extremity of the cantilever. The further the sphere is from the extremity, the greater is the haptic force that tends to pull the user to the predefined position [43]. Note that the insets representing the cantilever and the sphere are illustrations only. In the real setup only a top view is available, and the sphere is hidden by the cantilever. The haptic force is thus the only feedback that enables users to localize the sphere.

on the measurement of these oscillations has been proposed in [44], where virtual guides assist the user while aligning the cantilevers with the object and while closing the gripper (Fig. 6). 3D pick-and-place experiments of microspheres (diameter: $4-6 \mu \mathrm{m})$ validate the approach.

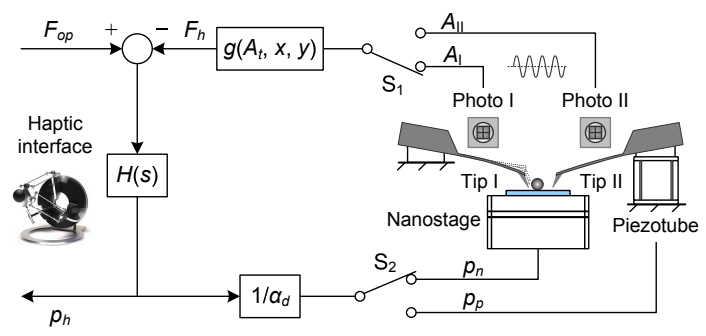

Fig. 6. Use of two AFM cantilevers to form a gripper: haptic feedback based on measurement of cantilever oscillations [44]

The complexity of the previous approach based on two AFM cantilevers to form a gripper, which makes it necessary to align each tip separately, makes it unsuitable for non-expert users. Classical grippers are better adapted to pick-and-place tasks, but they have one major drawback: they are usually sensor-deprived since the integration of sensors increases the complexity of the design and of the fabrication process. Vision is a commonly used solution for sensing; unfortunately, the low update rate of the frame-based acquisition process of current available cameras cannot ensure stable haptic feedback at the microscale level, where low inertia produces highly unreachable dynamic phenomena. A novel vision-based microrobotic system combining an asynchronous Address Event Representation silicon retina with a conventional frame-based camera is presented in [47]. Unlike frame-based cameras, recent artificial retinas transmit their outputs as a continuous stream of asynchronous temporal events, in a manner similar to the output cells of a biological retina. The reduction of redundant information enables high update rates. The temporal precision of the asynchronous silicon retina is used to provide 
a haptic feedback to assist users during manipulation tasks, whereas the frame-based camera is used to retrieve the position of the object that must be manipulated. This approach is validated through an experiment on teleoperating a sphere of around $50 \mu \mathrm{m}$ in diameter using a piezo-electric gripper in a pick-and-place task (Fig. 7).

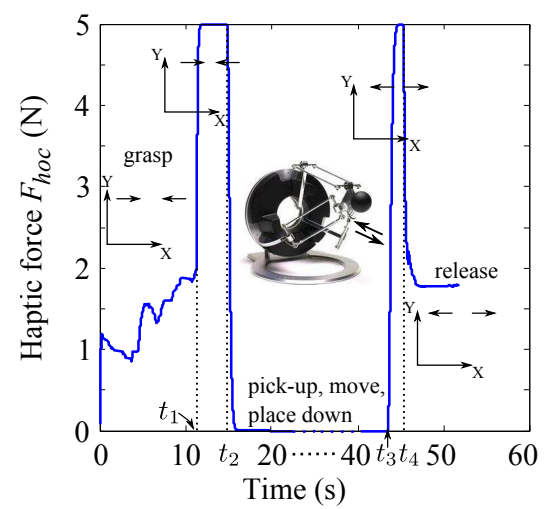

Fig. 7. Haptic feedback for a pick-and-place operation using a sensordeprived microgripper. An asynchronous Address Event Representation silicon retina and a conventional frame-based camera provide information about the relative positions of the tool and the object to compute the haptic force [47].

\section{Additional Assistance}

All the systems presented in the previous sections only provide haptic feedback based on available measurement. This is direct teleoperation. Two other types of teleoperation exist: virtual teleoperation where the user interacts with a simulator, and augmented teleoperation where he/she realizes a manipulation on a real object, but additional assistance is provided through the use of a simulator (Fig. 8).

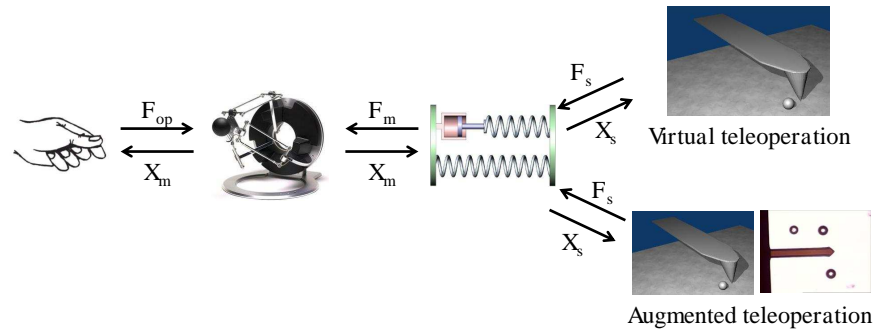

Fig. 8. Virtual and augmented teleoperation systems

\section{A. Virtual Teleoperation}

Virtual teleoperation has three main application fields which are education, training and evaluation. At this scale the objects and the tools are fragile, and the systems are highly sensitive to environmental conditions. Real systems are thus inappropriate for tests since it is not possible to guarantee the same experimental conditions for two trials.

Simulators have been developed for educational purposes. In [48], the benefit of haptic feedback and visual analogy for the comprehension of nanoscale phenomena is evaluated. A combination of these two modalities proves to be effective in teaching physics at the microscale to students.

Simulators can also prove to be efficient tools for training. Contrary to education where the goal is to teach the students unknown phenomena, training is dedicated to operators that work in the micromanipulation field. These simulators are then used by novice operators to learn technical gestures to perform a given assembly task, or by expert users to test different manipulation strategies. Virtual teleoperation systems have been developed to feel substrates geometries [49], or to simulate indentation tasks [23]. Some simulators can be adapted to the experimental conditions. For example, in [50] the geometry of the substrate is directly interpolated from real measurements. Several physical parameters can be tuned to change the physical properties such as friction. This ensures a realistic haptic rendering, which is necessary for training.

The simulators are also used for the definition of the most appropriate coupling schemes. Different haptic couplings are compared in [23]. Since the simulation guarantees the same experimental conditions, the performance of each control scheme can be evaluated.

\section{B. Augmented Teleoperation}

Augmented teleoperation systems make it possible to perform tasks on real objects, and benefit from additional information based on the simulator (Fig 9).

The first augmented teleoperated system is proposed in [51]. The manipulation is semi-teleoperated: the operator controls the overall operation, but some tasks are performed automatically [52], [53]. Users can thus concentrate on the main task, while the technical gestures are performed automatically.

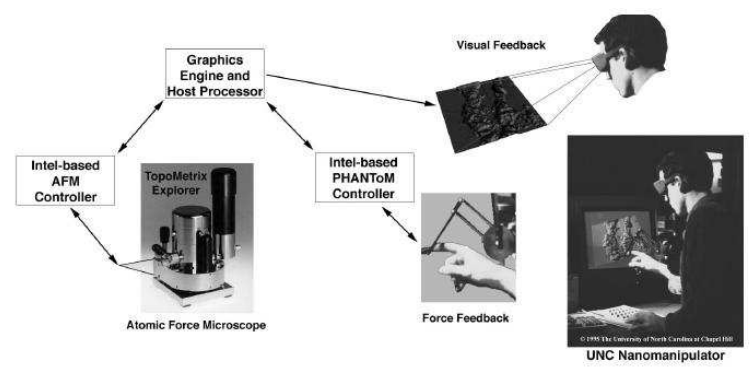

Fig. 9. The nanoManipulator offers semi-teleoperated augmented reality teleoperation [54]

Most augmented teleoperation systems propose a visual reconstruction of the scene. This is of utmost importance since on a microscale the visual feedback is limited. It is not possible to get a cheap real-time visual feedback of objects of less than hundreds of micrometers with depth information. The visual reconstruction of the scene makes it possible to add information (Fig. 10). It can be used to highlight physical phenomena such as the deformations applied on objects [55], [24]. Additional information can also be displayed to assist the user to perform a given task. In [42] the optimal path, as well 


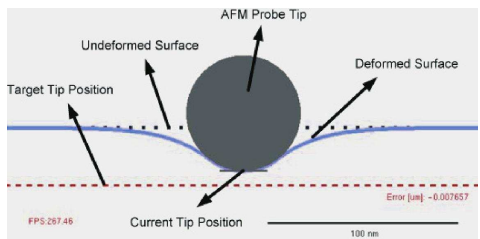

(a) Representation of the deformations of the substrate [24]

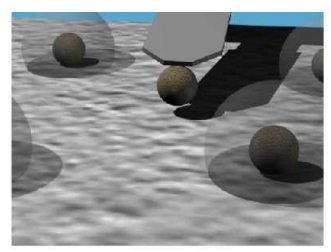

(b) Addition of virtual information to assist users to avoid obstacles [42]
Fig. 10. Visual reconstruction of the scene for augmented teleoperation systems

as areas that should be avoided to prevent collisions between the tool and the objects, are represented.

In the case of remote teleoperation, for example, if the user and the micromanipulation system are situated in geographically distant locations, the virtual reconstruction of the scene makes it possible to limit the load of the data transmitted. In [56] a 3D stereoscopic view of the scene is reconstructed based on the position of the object and the tool derived from images coming from a scanning electron microscope. Instead of transmitting full images, only two positions are sent between the two geographically distant sites to provide a real-time visual feedback (Fig. 11).

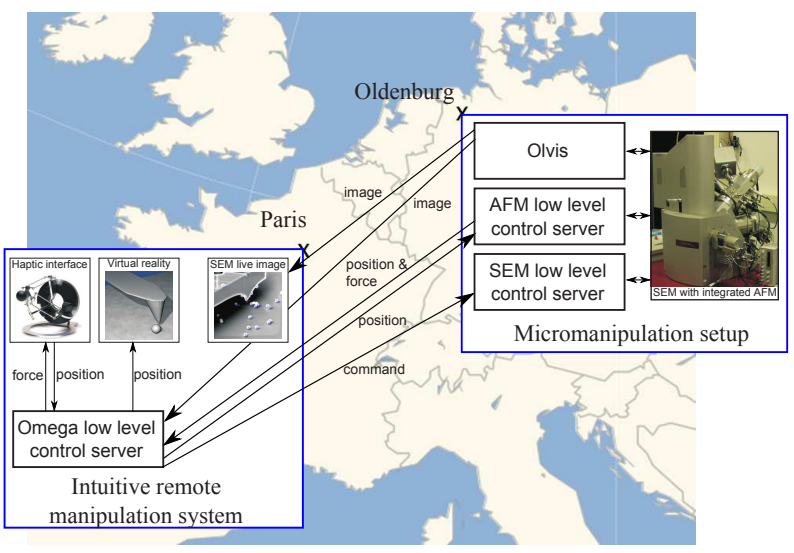

Fig. 11. Software architecture of a teleoperation system enabling micromanipulation from France of objects situated in Germany [56]. Information about the relative positions of the objects and the tool is derived from SEM images and transmitted to the remote teleoperation system. This is used to reconstruct a 3D virtual scene and to provide haptic feedback.

All these systems provide a haptic feedback. The visual reconstruction of the scene, which can use a simulator to provide a more realistic rendering, enhances the intuitiveness of the system. In addition, an audio display can be considered, as in [57] where an audio representation of the contact between a micro tactile sensor and the substrate is proposed. Audio feedback can also be combined with haptic feedback to enhance the assistance provided to users [58], [59]. However, its use remains rather limited on the microscale.

\section{CONClusion AND Future Directions}

Since the first works developed in the 1990s, haptic feedback teleoperation systems have shown drastic changes. The first AFM-based systems made it possible to "touch the microworld" by providing the feeling of the substrate topology. Detailed analyses of the haptic coupling scheme performance made it possible to improve both stability and transparency. Based on the determination of strategies to provide $2 \mathrm{D}$ or $3 \mathrm{D}$ haptic feedback despite the lack of force and position sensors, more complex applications have been proposed. Vision-based haptic feedback makes it possible to use a wide range of sensor-deprived tools, and in particular the microgrippers that are present in most microassembly platforms. Augmented teleoperation systems that use simulators to derive additional information improve the assistance provided to operators. Virtual teleoperation systems enable the training of users, as well as testing manipulation strategies.

Based on all these developments on haptic feedback teleoperation for micromanipulation and virtual reality assistance, this field is mature for microassembly tasks involving objects as small as 1 to 10 micrometers. The strategies developed for research laboratory needs are now ready for the transfer of technology. The solutions reviewed throughout this paper can be integrated to fulfill the requirements of an industrial microassembly system. This can benefit small-scale industrial projects where complex assembly tasks of objects whose size ranges from a few micrometers to several hundreds of micrometers must be performed. This leverages the user expertise by enabling operators to concentrate on critical issues of the manipulation while assisting them to perform the assembly.

Several future directions can be foreseen to increase the effectiveness of such systems. In particular, specific haptic interfaces should be developed. Most of the works presented in this paper use commercially available haptic interfaces and none of them are dedicated to microassembly tasks. To increase the effectiveness of such systems, specific interfaces, with adapted design and haptic feedback specifications, should be conceived. Large-scale user-based tests performed on industrial end users should be performed to ensure a perfect match between the haptic feedback and the industrial needs. Most of the works presented in this paper deals with AFMbased systems, as they were historically widely used. Future developments should concentrate on providing haptic feedback for lower cost user friendly tools, to efficiently address the microassembly industrial projects.

\section{ACKNOWLEDGMENT}

This work was supported by the French National Agency of Research, through the NANOROBUST project.

\section{REFERENCES}

[1] M. Gauthier and S. Régnier, Robotic Micro-Assembly. Wiley-IEEE Press, 2010

[2] S. Régnier and N. Chaillet, Microrobotics for Micromanipulation. J. Wiley \& Sons, 2010.

[3] H. Xie, C. D. Onal, S. Régnier, and M. Sitti, Atomic Force Microscopy Based Nanorobotics, modelling, simulation, setup building and experiments series ed., ser. Springer Tracts in Advanced Robotics, 2011, vol. 71.

[4] T. C. Duc, G. Lau, J. F. Creemer, and P. M. Sarro, "Electrothermal microgripper with large jaw displacement and integrated force sensors," Journal of Microelectromechanical Systems, vol. 17, no. 6, pp. 15461555, 2008. 
[5] L. Wang, L. Ren, J. Mills, and W. Cleghorn, "Automated 3-D micrograsping tasks performed by Vision-Based control," IEEE Transactions on Automation Science and Engineering, vol. 7, no. 3, pp. $417-426$, 2010.

[6] C. Onal and M. Sitti, "Visual Servoing-Based autonomous 2-D manipulation of microparticles using a nanoprobe," IEEE Transactions on Control Systems Technology, vol. 15, no. 5, 2007.

[7] S. Bargiel, K. Rabenorosoa, C. Clévy, C. Gorecki, and P. Lutz, "Towards micro-assembly of hybrid MOEMS components on reconfigurable silicon free-space micro-optical bench." Journal of Micromechanics and Microengineering, vol. 20, no. 4, p. 20 pages, 2010.

[8] A. Ferreira and C. Mavroidis, "Virtual reality and haptics for nanorobotics," IEEE Robotics and Automation Magazine, vol. 13, no. 3, pp. 78-92, 2006.

[9] J. E. Colgate, "Robust impedance shaping telemanipulation." IEEE Transcations on Robotics and Automation, vol. 9, no. 4, pp. 374-384, 1993.

[10] Y. Yokokohji, N. Hosotani, and T. Yoshikawa, "Analysis of maneuverability and stability of micro-teleoperation systems," in IEEE International Conference on Robotics and Automation, 1994, pp. 237-243.

[11] Q. Zhou, P. Kallio, and H. N. Koivo, "Fuzzy control system for microtelemanipulation," in Intelligent Automation and Control: Recent Trends in Development and Applications, 1996, pp. 817-822.

[12] F. Fukuda, K. Tanie, and T. Mitsuoka, "A new method of master-slave type of teleoperation for a micro-manipulator system," in IEEE Micro Robots and Teleoperators Workshop, 1987, pp. 204-208.

[13] S. E. Salcudean and J. Yan, "Towards a force-reflecting motion-scaling system for microsurgery," in IEEE International Conference on Robotics and Automation, 1994, pp. 2296-2301.

[14] P. Kallio and H. N. Koivo, "A 1 d.o.f. mini-telemanipulator: Design and control," in International Conference on Robotics and Manufacturing, 1995, pp. 192-198.

[15] Y. Hatamura and H. Morishita, "Direct coupling system between nanometer world and human world," in IEEE International Conference on Micro Electro Mechanical Systems, 1990, pp. 203-208.

[16] R. Hollis, S. Salcudean, and D. Abraham, "Toward a tele-nanorobotic manipulation system with atomic scale force feedback and motion resolution," in IEEE International Conference on Micro Electro Mechanical Systems, 1990, pp. 115-119.

[17] M. Sitti and H. Hashimoto, "Macro to nano tele-manipulation through nanoelectromechanical systems," in IEEE Industrial Electronics Society, 1998, pp. 98-103.

[18] _ - "Teleoperated touch feedback from the surfaces at the nanoscale: modeling and experiments," IEEE/ASME Transactions on Mechatronics, vol. 8, no. 2, pp. 287-298, 2003.

[19] G. Venture, D. S. Haliyo, A. Micaelli, and S. Régnier, "Force-feedback micromanipulation with inconditionnally stable coupling," International Journal of Micromechatronics, special issue on Micro-handling, vol. 3, no. 3, pp. 307-327, 2006.

[20] M. Sitti and H. Hashimoto, "Tele-nanorobotics using atomic force microscope," in IEEE/RSJ International Conference on Intelligent Robots and Systems, vol. 3, 1998, pp. 1739-1746.

[21] A. Bolopion, B. Cagneau, S. Haliyo, and S. Régnier, "Analysis of stability and transparency for nanoscale force feedback in bilateral coupling," Journal of Micro - Nano Mechatronics, no. 4, pp. 145-158, 2009.

[22] F. Llewellyn, "Some fundamental properties of transmission systems," IRE, vol. 40, no. 3, pp. 271-283, 1952.

[23] S.-G. Kim and M. Sitti, "Task-based and stable telenanomanipulation in a nanoscale virtual environment," IEEE Transactions on Automation Science and Engineering, vol. 3, no. 3, pp. 240-247, 2006.

[24] C. D. Onal and M. Sitti, "A scaled bilateral control system for experimental one-dimensional teleoperated nanomanipulation," The International Journal of Robotics Research, vol. 28, no. 4, pp. 484-497, 2009.

[25] M. Boukhnifer and A. Ferreira, "Wave-based passive control for transparent micro-teleoperation system," Robotics and Autonomous Systems, vol. 54, no. 7, pp. $601-615,2006$.

[26] - , $\mathrm{H}_{\infty}$ loop shaping bilateral controller for a two-fingered telemicromanipulation system," IEEE Transactions on Control Systems Technology, vol. 15, no. 5, pp. 891-905, 2007.

[27] S. Fahlbusch and S. Fatikow, "Force sensing in microrobotic systemsan overview," in International Conference on Electronics, Circuits and Systems, vol. 3, 1998, pp. 259-262 vol.3.

[28] F. Beyeler, S. Muntwyler, and B. J. Nelson, "A six-axis MEMS force torque sensor with micro-newton and nano-newtonmeter resolution," Journal of Microelectromechanical Systems, vol. 18, no. 2, pp. 433441, 2009.
[29] J. Zhang, G. Li, and N. Xi, "Modeling and control of active end effector for the AFM based nano robotic manipulators," in IEEE International Conference on Robotics and Automation, 2005, pp. 163-168.

[30] L. Liu, N. Jiao, X. Tian, Z. Dong, N. Xi, W. Li, and Y. Wang, "Development of a haptic user interface for surface sensing and nanomanipulation based on atomic force microscope," in IEEE International Conference on Nano/Micro Engineered and Molecular Systems, 2006, pp. 900-904.

[31] C. D. Onal and M. Sitti, "Teleoperated 3-D force feedback from the nanoscale with an atomic force microscope," IEEE Transactions on nanotechnology, vol. 9, no. 1, pp. 46-54, 2010.

[32] K. Kim, X. Liu, Y. Zhang, and Y. Sun, "Micronewton force-controlled manipulation of biomaterials using a monolithic MEMS microgripper with two-axis force feedback," in IEEE International Conference on Robotics and Automation, 2008, pp. 3100-3105.

[33] [Online]. Available: http://www.femtotools.com

[34] B. Lopez-Walle, M. Gauthier, and N. Chaillet, "Principle of a submerged freeze gripper for microassembly," IEEE Transactions on Robotics, vol. 24, no. 4, pp. 897-902, 2008.

[35] K. N. Andersen, D. H. Petersen, K. Carlson, K. Molhave, O. Sardan, A. Horsewell, V. Eichhorn, S. Fatikow, and P. Boggild, "Multimodal electrothermal silicon microgrippers for nanotube manipulation," IEEE Transactions on Nanotechnology, vol. 8, no. 1, pp. 76-85, 2009.

[36] Y. Zhou, B. Nelson, and B. Vikramaditya, "Fusing force and vision feedback for micromanipulation," in IEEE International Conference on Robotics and Automation, vol. 2, 1998, pp. 1220-1225.

[37] M. Greminger and B. Nelson, "Vision-based force measurement," IEEE Transactions on Pattern Analysis and Machine Intelligence, vol. 26, no. 3, pp. 290-298, 2004.

[38] A. N. Reddy, D. K. Sahu, N. Maheswari, and G. K. Ananthasuresh, "Miniature compliant grippers with vision-based force-sensing," IEEE Transactions on Robotics, vol. 26, no. 5, pp. 867-877, 2010.

[39] M. Greminger, G. Yang, and B. Nelson, "Sensing nanonewton level forces by visually tracking structural deformations," in IEEE International Conference on Robotics and Automation, vol. 2, 2002, pp. 19431948.

[40] D. Cappelleri, G. Piazza, and V. Kumar, "A two dimensional visionbased force sensor for microrobotic applications," Sensors \& Actuators: A. Physical, vol. 171, no. 2, pp. 340-351, 2011.

[41] D. Haliyo, S. Régnier, and J. Guinot, "[mu] mad, the adhesion based dynamic micro-manipulator," European Journal of Mechanics-A/Solids, vol. 22 , no. 6, pp. 903-916, 2003.

[42] M. Ammi and A. Ferreira, "Robotic assisted micromanipulation system using virtual fixtures and metaphors," in IEEE International Conference on Robotics and Automation, 2007, pp. 454-460.

[43] A. Bolopion, B. Cagneau, and S. Régnier, "2d micro teleoperation with force feedback," in IEEE/RSJ International Conference on Intelligent Robots and Systems, 2009, pp. 3265-3270.

[44] A. Bolopion, H. Xie, S. Haliyo, and S. Régnier, "Haptic teleoperation for 3d microassembly of spherical objects," IEEE/ASME Transaction on Mechatronics, 2010.

[45] H. Xie and S. Régnier, "Development of a flexible robotic system for multiscale applications of micro/nanoscale manipulation and assembly," IEEE/ASME Transactions Mechatronics, vol. 16, no. 2, pp. 266-276, 2011.

[46] H. Xie, S. Haliyo, and S. Régnier, "Parallel imaging/manipulation force microscopy," Applied Physics Letters, vol. 94, p. 153106, 2009.

[47] Z. Ni, A. Bolopion, J. Agnus, R. Benosman, and S. Régnier, "Asynchronous event-based visual shape tracking for stable haptic feedback in microrobotics," IEEE Transactions on Robotics, 2012, in press.

[48] G. Millet, A. Lécuyer, J. Burkhardt, D. Haliyo, and S. Régnier, "Improving perception and understanding of nanoscale phenomena using haptics and visual analogy," in Eurohaptics. Springer-Verlag, 2008, pp. $847-856$.

[49] S. Grange, F. Conti, P. Helmer, P. Rouiller, and C. Baur, "The Delta Haptic Device as a nanomanipulator," in SPIE Microrobotics and Microassembly III, 2001.

[50] W. Vogl, B. K.-L. Ma, and M. Sitti, "Augmented reality user interface for an atomic force microscope-based nanorobotic system," IEEE Transactions on Nanotechnology, vol. 5, no. 4, pp. 397-406, 2006.

[51] R. Taylor II, J. Chen, S. Okimoto, N. Llopis-Artime, V. L. Chi, F. P. Brooks Jr., M. Falvo, S. Paulson, P. Thiansathaporn, D. Glick, S. Washburn, and R. Superfine, "Pearls found on the way to the ideal interface for scanned-probe microscopes," in 8th conference on Visualization, 1997, pp. 467-ff.

[52] M. Falvo, G. Clary, A. Helser, S. Paulson, R. Taylor II, V. Chi, F. Brooks Jr., S. Washburn, and R. Superfine, "Nanomanipulation experiments 
exploring frictional and mechanical properties of carbon nanotubes," Journal of Microscopy and Microanalysis, vol. 4, pp. 504-512, 1999.

[53] M. Guthold, M. Falvo, W. Matthews, S. Paulson, S. Washburn, D. Erie, R. Superfine, F. Brooks Jr., and R. Taylor II, "Controlled manipulation of molecular samples with the nanoManipulator," IEEE/ASME Transactions on Mechatronics, vol. 5, no. 2, pp. 189-198, 2000.

[54] R. Taylor II, "Programming force feedback devices in computer graphics systems," in Course notes for "Programming Virtual Worlds", SIGGRAPH, 1997.

[55] L. Fok, Y. Liu, and W. Li, "Modeling of nanomanipulation with an integrated teleoperated system," in IEEE International Conference on Robotics and Biomimetics, 2005, pp. 83-88.

[56] A. Bolopion, C. Dahmen, C. Stolle, S. Haliyo, S. Régnier, and S. Fatikow, "Vision based haptic feedback for remote micromanipulation in a sem environment," International Journal of Optomechatronics, 2012 , in press.

[57] Y. Murayama, C. E. Constantinou, and S. Omata, "Intuitive micromanipulation with haptic audio feedback," in IEEE International Conference on Computer and Information Technology, Sep. 2004, pp. 907- 911.

[58] A. Luciani, D. Urma, S. Marlière, and J. Chevrier, "Presence: The sense of believability of inaccessible worlds," Computers \& Graphics, vol. 28, pp. 509-517, 2004.

[59] F. Marchi, D. Urma, S. Marliere, J. L. Florens, A. Besancon, J. Chevrier, and A. Luciani, "Educational tool for nanophysics using multisensory rendering," in World Haptics Conference, vol. 0, 2005, pp. 473-474.

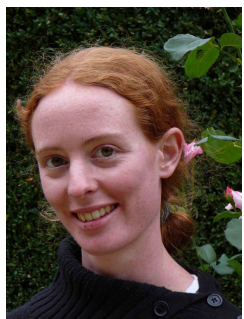

Aude Bolopion received her $\mathrm{Ph} . \mathrm{D}$. degree in robotics in 2010 from the University of Pierre \& Marie Curie, Paris, France.

She is currently a CNRS researcher at the FEMTO-ST Institute, Besancon, France. She has been a member of the Biomedical Micro Nano Robotics team since 2011. From 2007 and 2011 she was a member of the ISIR micromanipulation team. Her research interests are focused on teleoperation and haptic feedback at the nanoscale, and on non contact micromanipulation.

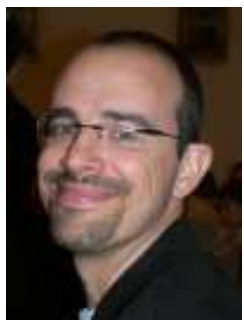

Stéphane Régnier received his $\mathrm{PhD}$ degree in $\mathrm{Me}$ chanics and Robotics from the University of Pierre \& Marie Curie, Paris, France in 1996.

$\mathrm{He}$ is currently Professor at the Institute of Intelligent Systems and Robotics (ISIR), University of Pierre \& Marie Curie, Paris, France. He has been head of the ISIR micro/nanorobotics team since 2001. His research interests are focused on micro and nano manipulation, teleoperation and haptic feedback at the nanoscale, micromechatronics and biological cell characterization. 